\title{
Depolarization ratio measurement using single photomultiplier tube in micropulse lidar
}

\author{
P. K. Dubey, ${ }^{\text {a) }}$ S. L. Jain, B. C. Arya, and Pavan S. Kulkarni \\ Radio and Atmospheric Science Division, National Physical Laboratory, New Delhi 110012 India
}

(Received 12 February 2009; accepted 28 April 2009; published online 22 May 2009)

\begin{abstract}
The conventional dual polarization micropulse lidar uses two separate photomultiplier tubes (PMT) to detect both the copolarized and cross-polarized beam. The prominent sources of error in the depolarization ratio measurement are mismatch in PMT, improper selection of discriminator threshold and unequal PMT high voltage. In the present work a technique for the measurement of lidar depolarization ratio using only one PMT sensor has been developed. The same PMT detects both copolarized and cross-polarized lidar backscatter. A stepper motor is used along with the mirrors to bring both the received polarization signals over the PMT window. Application of the same PMT minimizes the error caused in the depolarization ratio measurement due to error in photon counting of an individual channel. The design description of this technique along with the preliminary results depicting its functionality has been mentioned in this article. () 2009 American Institute of Physics. [DOI: 10.1063/1.3137934]
\end{abstract}

\section{INTRODUCTION}

Light detection and ranging (lidar) deals with the profiling of atmosphere and has largely contributed to our knowledge of Earth's atmosphere during past decades. ${ }^{1,2}$ Lidar measurements provide vertical profiling of aerosols with high temporal and spatial resolution which makes it possible to observe the atmosphere at ambient conditions and permit a clear separation of optical properties of boundary layer particles. Micropulse lidar (MPL) is an active remote sensing instrument that emits low energy laser pulses toward the atmosphere or target and measures the back scattered signal. MPL is being widely used to monitor meteorological parameters and atmospheric constituents. ${ }^{3-5}$

Polarization MPL has been used to study various phenomenon in the atmosphere, such as hydrometeors, ${ }^{6}$ clouds, ${ }^{7}$ volcanic aerosols, ${ }^{8}$ and polar stratospheric clouds. ${ }^{9,10}$ The space-based lidars such as CALIPSO include a crosspolarization receiver channel because of the valuable information that it provides. ${ }^{9}$ The value of the depolarization ratio of spherical particles such as liquid droplets is zero for backscattering, based on the Mie-scattering theory; enhancement of the depolarization ratio can arise from scattering by nonspherical particles such as solid particles or by multiple scattering. ${ }^{10}$ It became apparent that in comparison to microwave depolarization from nonspherical particles (typically smaller than the incident wavelength) the laser depolarization (from particles larger than the wavelength) was considerably stronger, suggesting that polarization lidar has a promising future for the study of aerosols and the particles in clouds. The basic polarization lidar application involves the transmission of a linearly polarized laser pulse and the detection via a beam splitter of the orthogonal and parallel

\footnotetext{
${ }^{\text {a) }}$ Author to whom correspondence should be addressed. Electronic mail: premkdubey@gmail.com.
}

planes of polarization of the backscattered light. The ratio of these two signals is referred to as the linear depolarization ratio $^{3,11}(\delta)$,

$$
\delta(z)=\frac{P_{\perp}(z)}{P_{I I}(z)} .
$$

Uncertainties in lidar depolarization measurements stem from various sources, but are basically related to (a) errors in accounting for the differences in the optical and electronic gains of the two channels (b) polarization purity of the laser pulse and (c) the alignment between the polarization plane of the laser and that of the polarizer(s) in the detectors. ${ }^{3,12}$ In the receivers, having photon counting technique for the reconstruction of the received signal, the error in receiver gain may occur due to mismatch in photomultiplier tube (PMTs), variation in HV supply, mismatch in quantum efficiency and discriminator threshold selection errors. The mismatch in PMTs and their parameters can be greatly improved by using the PMTs of same model. However improper selection of PMT high voltage supply and discriminator threshold is still the source of error even in matched PMTs.

Keeping the above constraints in mind, a new measurement technique has been developed which minimizes the error in the de polarization ratio measurement due to the differences in the electronic gains of the two channels. The technique uses only one PMT (fixed) to receive both the input channels i.e., perpendicular and parallel polarization. A microstepping motor along with mirror has been used in half step mode to select the polarization. An alternate scanning method has been used to record $P$ and $S$ polarization data. Since only one PMT is being used, the error caused by two separate channels and improper selection of discriminator threshold has been minimized. The functionality of the technique along with some preliminary results has been discussed in this article. 


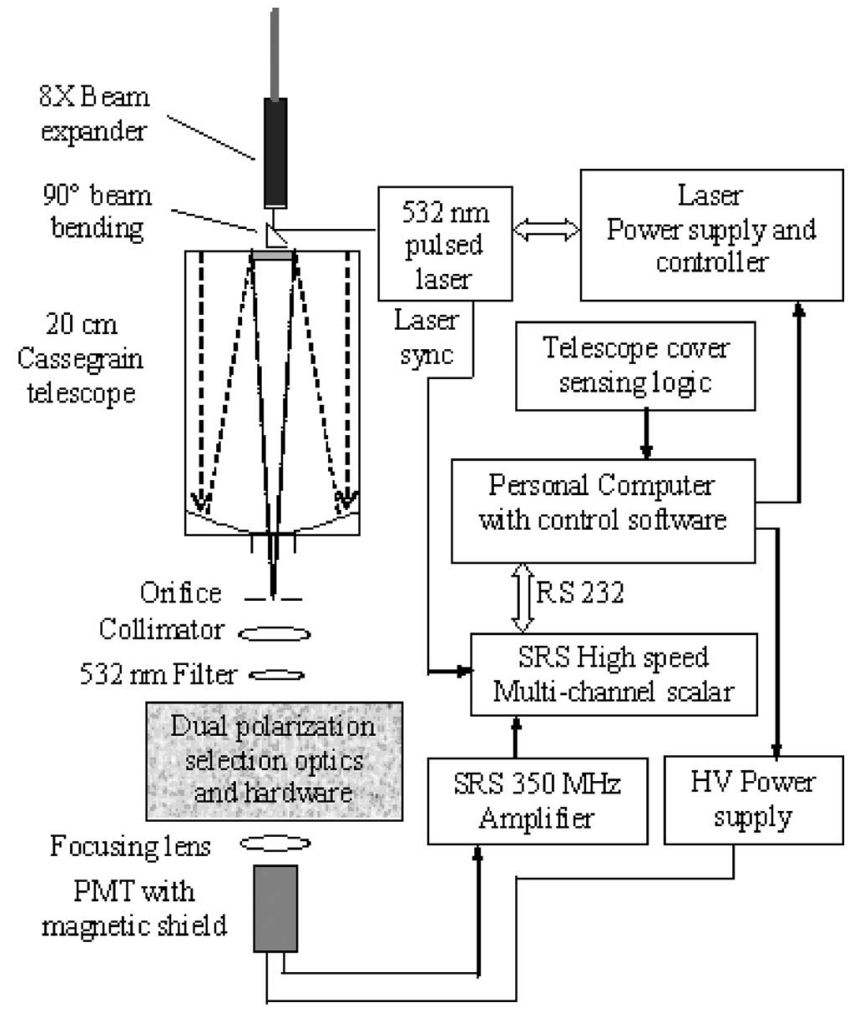

FIG. 1. Schematic of the lidar system developed at NPL with improved dual polarization detection system.

\section{EXPERIMENTAL}

\section{A. The micropulse lidar}

A portable MPL system was developed at National Physical Laboratory, New Delhi $\left[28^{\circ} 65^{\prime} \mathrm{N}, 77^{\circ} 28^{\prime} \mathrm{E}, 220 \mathrm{~m}\right.$ above sea level (ASL)] and is functional since December 2007. The MPL system is being used to study the atmospheric boundary layer $(\mathrm{ABL})$ and clouds. It is kept in 4 $\times 3 \mathrm{~m}^{2}$ air-conditioned room. An opening of $45 \times 45 \mathrm{~cm}^{2}$ in the roof of the room is used to send the laser beam vertically up in the atmosphere and the backscattered signal is collected through a $20 \mathrm{~cm}$ cassegrain receiving telescope. ${ }^{13}$

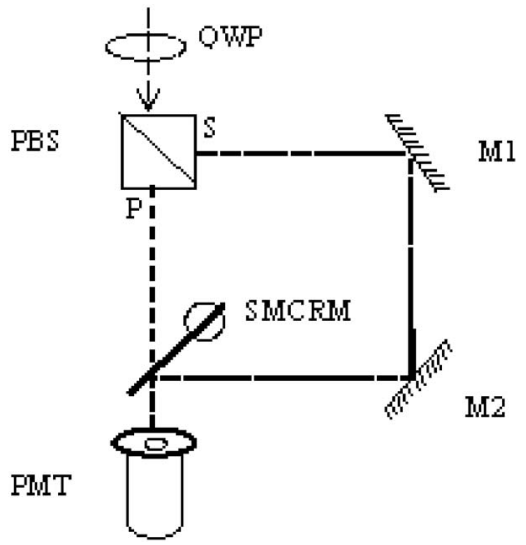

FIG. 2. Schematic depicting reception of $P$ and $S$ polarizations using single PMT

Figure 1 shows the schematic of the MPL with an added dual polarization detection system. Table I lists important specification of this MPL.

\section{B. Dual polarization detection by single PMT}

The dual polarization detection system using single PMT has been successfully designed and added in the lidar system. Figure 2 shows the scheme of the developed dual polarization detection system. The polarization beam splitter ${ }^{14}$ (CVI laser: PBS-532-050) separates the received back scattered signal into copolarized $(P)$ and cross-polarized $(S)$ components. Fixed mirrors M1 and M2 are used to bring the cross-polarized signal beam on the reflecting surface of the stepper motor controlled rotating mirror (SMCRM). This polarization selection mirror (SMCRM) has the reflecting front surface with absorbing coating at the back. This mirror is mounted on a microstepping stepper motor (taken from an old 5.25 in. floppy drive). The stepping motor is driven in half step mode to achieve a step resolution of $0.9^{\circ}$. The conventional metal oxide semiconductor field effect transistor (MOSFET) (IRF 540) based circuit has been used to drive the stepper motor.

TABLE I. Important specifications of the MPL.

\begin{tabular}{|c|c|c|}
\hline Section & Parameter & Specification \\
\hline Transmitter & $\begin{array}{c}\text { Laser type } \\
\text { Wavelength } \\
\text { Output pulse energy } \\
\text { Pulse duration } \\
\text { Pulse repetition rate } \\
\text { Polarization } \\
\text { Beam divergence (after beam expander) }\end{array}$ & $\begin{array}{l}\text { Diode pumped solid state } Q \text {-switched Nd:YAG laser } \\
532 \mathrm{~nm} \\
4 \mu \mathrm{J} \\
900 \mathrm{pS} \\
7.5 \mathrm{kHz} \\
\text { Linear } \\
150 \mu \mathrm{rad}\end{array}$ \\
\hline Receiver & $\begin{array}{l}\text { Telescope type } \\
\text { Telescope diameter } \\
\text { Field of view } \\
\text { Optical sensor }\end{array}$ & $\begin{array}{c}\text { Schmidt cassegranian } \\
20 \mathrm{~cm} \\
<300 \mu \mathrm{rad} \\
\text { PMT, Hamamatsu: R647 }\end{array}$ \\
\hline Signal processing and electronics & $\begin{array}{l}\text { Data acquisition technique } \\
\text { Bin resolutions (selectable) } \\
\text { Range (selectable) }\end{array}$ & $\begin{array}{c}\text { (single photon counting) } \\
0.75 \mathrm{~m}, 6 \mathrm{~m}, 12 \mathrm{~nm}, 24 \mathrm{~m} \text {, and } 48 \mathrm{~m} \\
1.5 \mathrm{~km}, 3 \mathrm{~km}, 6 \mathrm{~km} \text {, and } 12 \mathrm{~km}\end{array}$ \\
\hline Control software & $\begin{array}{l}\text { Software developed in visual basic } \\
\text { under WINDOwS XP environment }\end{array}$ & $\begin{array}{l}\text { Raw, background, and dark count acquisition and real time } \\
\text { acquisition and display of backscatter in } 1 \text { min interval }\end{array}$ \\
\hline
\end{tabular}




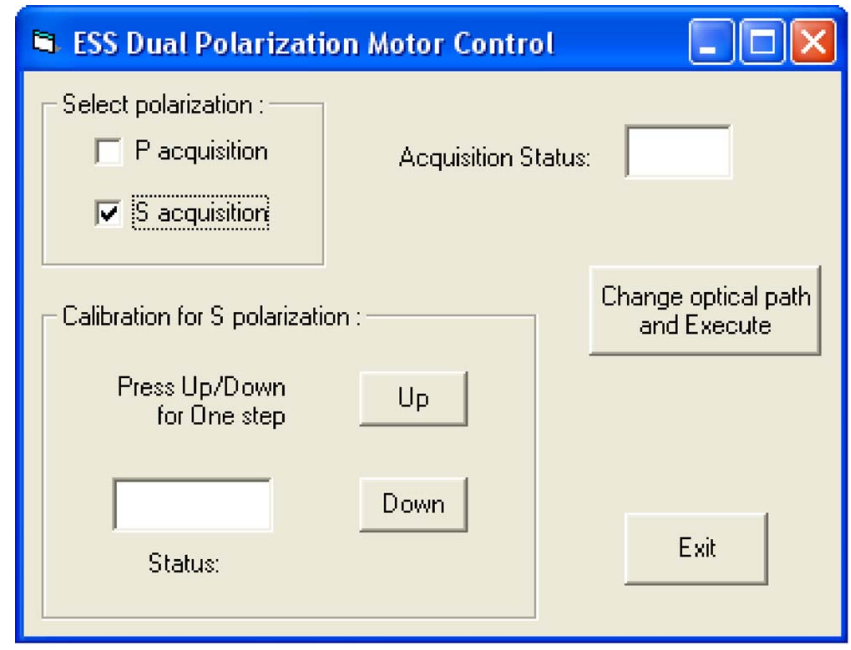

FIG. 3. (Color online) The GUI for the $S$ polarization calibration.

The stepping motor is controlled through the developed lidar software, using parallel printer port of the computer. Lower four bits of the port are used to drive the respective MOSFETS which in turn energizes the coils of the motor. The conventional bit combination is used to drive the motor in half step mode. The stepper motor coils are kept energized even after achieving the selected position $(P$ or $S)$ so that the position can be held with force.

The alignment software has also been developed in visual basic through which the motor is controlled and aligned for $S$ polarization reception. The Graphical User Interface (GUI) of the motor control software is shown in Fig. 3. The SMCRM is rotated by a step by using step up and down control of the GUI. The $S$ beam is aligned for reception by making the absorbing surface tilted toward the $P$ beam and approximately making incidence angle of $45^{\circ}$ with the incoming $P$ beam. This $P$ beam is absorbed by the absorbing surface. The stepping motor is adjusted for a step in either

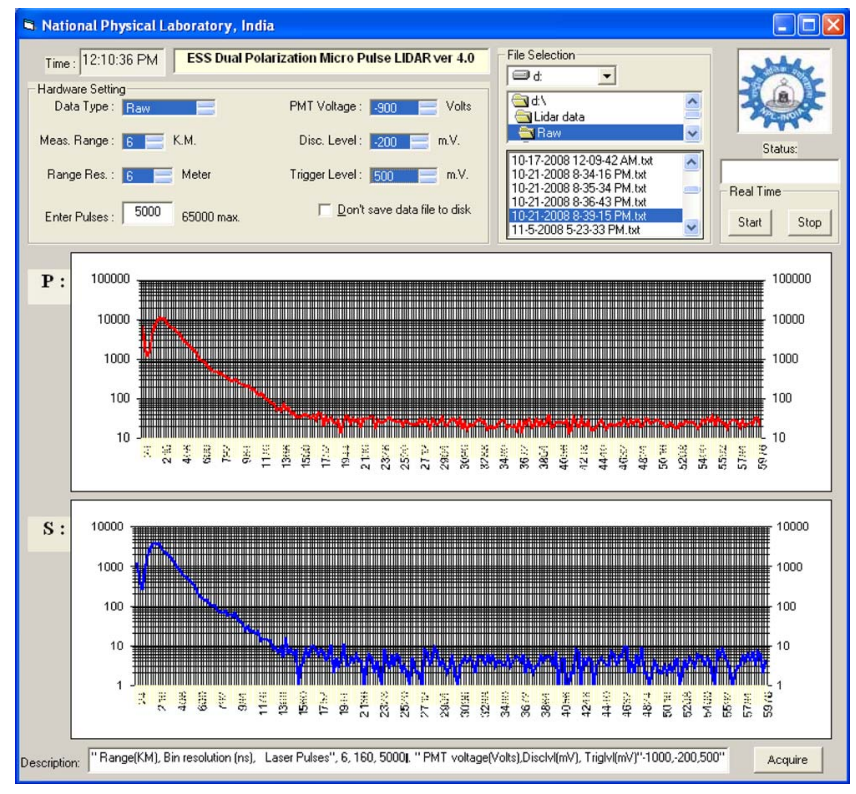

FIG. 4. (Color online) GUI of the developed control software; showing $P$ and $S$ components for the range of $6 \mathrm{~km}$.

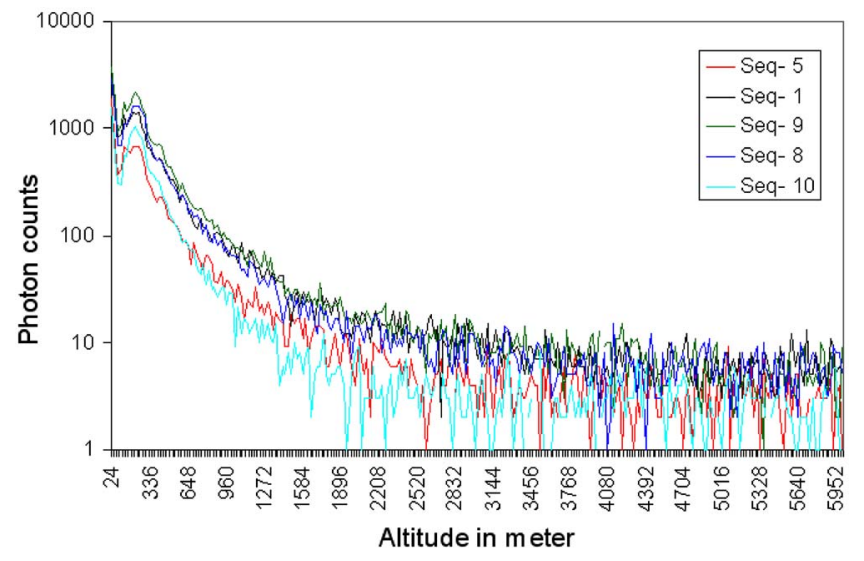

FIG. 5. (Color online) $S$ polarization signal received during its alignment by rotating the SMCRM at $0.9^{\circ}$ step.

direction so that maximum signal peak can be observed. This is considered as the $S$ state and the configuration (states of coil) is stored in a file in the computer. Now SMCRM is rotated by 45 steps $\left(40.5^{\circ}\right)$ anticlockwise, which clears the path of $P$ beam coming from PBS to reach PMT. This is considered as the $P$ state and the data acquired is of $P$ polarization. The SMCRM reflecting surface is not exactly in perpendicular with the incoming $S$ signal, rather while acquiring $P$ signal $S$ is diverted out of the incoming path. Thus, avoids back reflection of $S$ signal at the beam splitter.

\section{Technical considerations and limitations}

In lidar technology, as the backscattered signal received at a single transmitted laser pulse is very weak so it is almost difficult to understand the variations in the received signal. This is overcome by transmitting large number of laser pulses and integrating their individual responses at the receiver, which in turn improves the statistical precision. The entire atmosphere covered by the receiver field of view is assumed to be stable during this entire acquisition. The advent of high repetition rate pulsed lasers ${ }^{15,16}$ and very high speed photon counting boards ${ }^{17,18}$ makes it possible to integrate $10^{6}$ of such pulses in few seconds. The dual polarization detection technique explained here also assumes that the atmosphere is stable during the acquisition of both $P$ and $S$ polarization profiles.

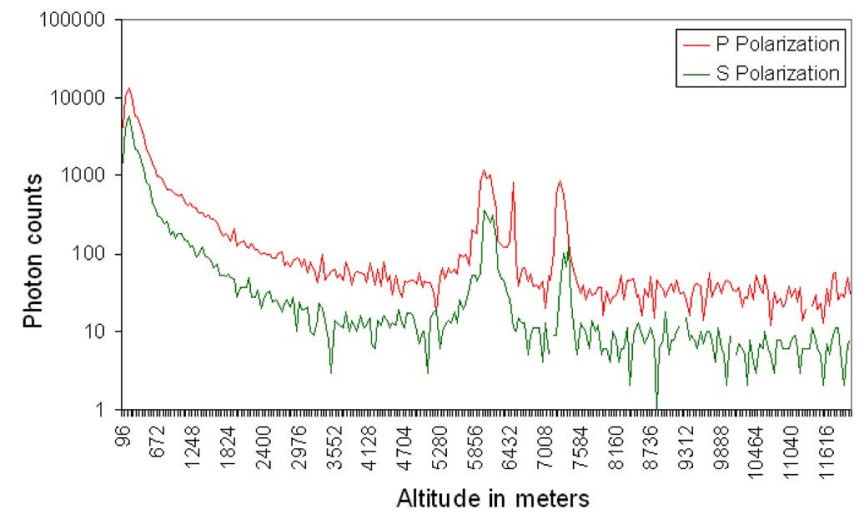

FIG. 6. (Color online) Lidar backscattered $P$ and $S$ data received on 22 January 2009 at 22.50 local time over the experimental site. 


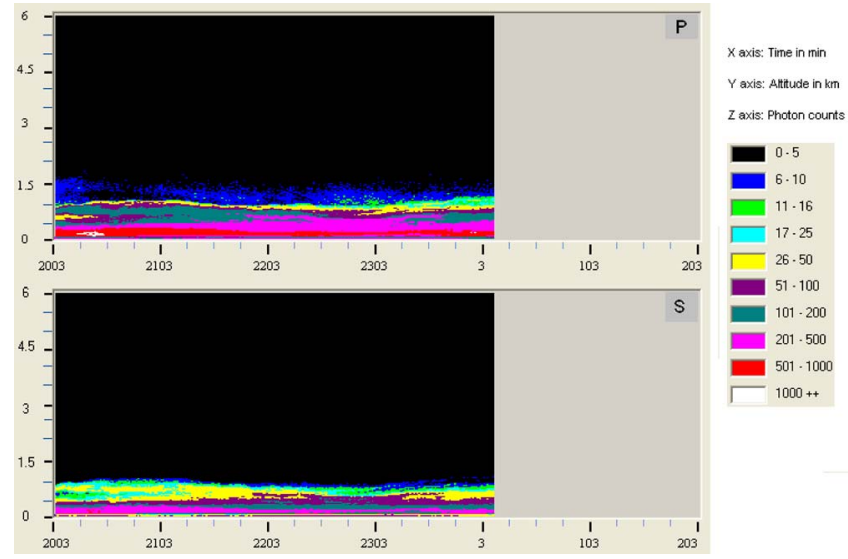

FIG. 7. (Color online) Lidar backscattered raw $P$ and $S$ data acquired on 16 October 2008 night time.

\section{Developed dual polarization MPL software}

The entire control software for the dual polarization MPL (DMPL) has been developed in Visual Basic (some functions of visual $\mathrm{C}$ is included in the software). The GUI of this software is shown in Fig. 4. All the lidar parameters can be set through this GUI including the PMT gain. A wide range of PMT voltage can be selected from the developed software and the discriminator threshold is selected according to the set voltage for optimum performance. The data acquired for both the polarization ( $P$ and $S$ polarizations) is displayed on screen and also the data file is stored in the disk for further processing. The software also supports an unattended autonomous mode of operation.

In autonomous mode, the DPMPL continuously acquires data at the set parameters (as shown in Fig. 4) at $1 \mathrm{~min}$ interval and store it into the computer memory. The software also has facility to plot three-dimensional raster of individual polarizations, which displays the temporal variations of atmospheric dynamics for the acquired time duration. In addition to this, the software also has facility to compute and plot real time linear depolarization ratio from the data stored in the computer.

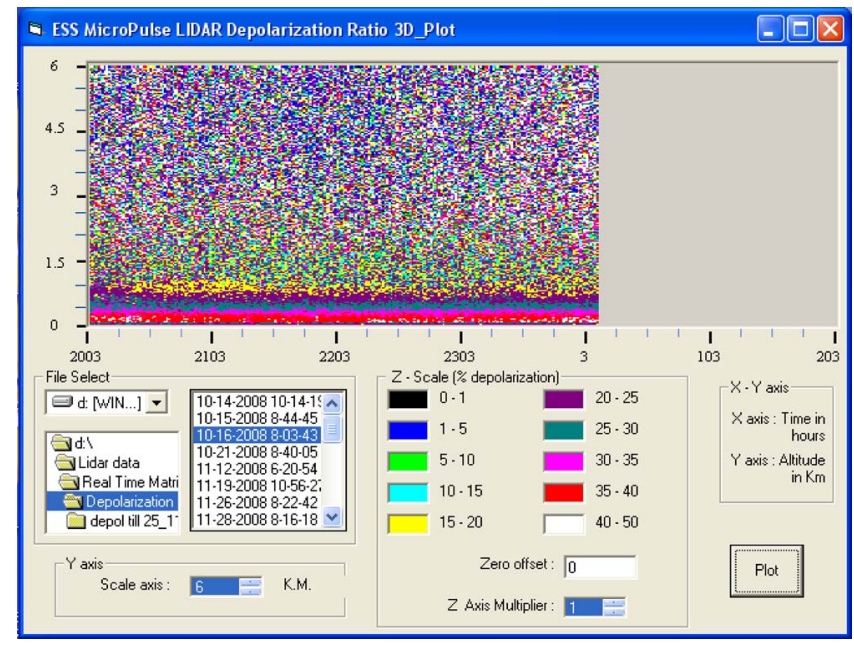

FIG. 8. (Color online) Typical depolarization profile extracted from data acquired on 16 October 2008 night time.

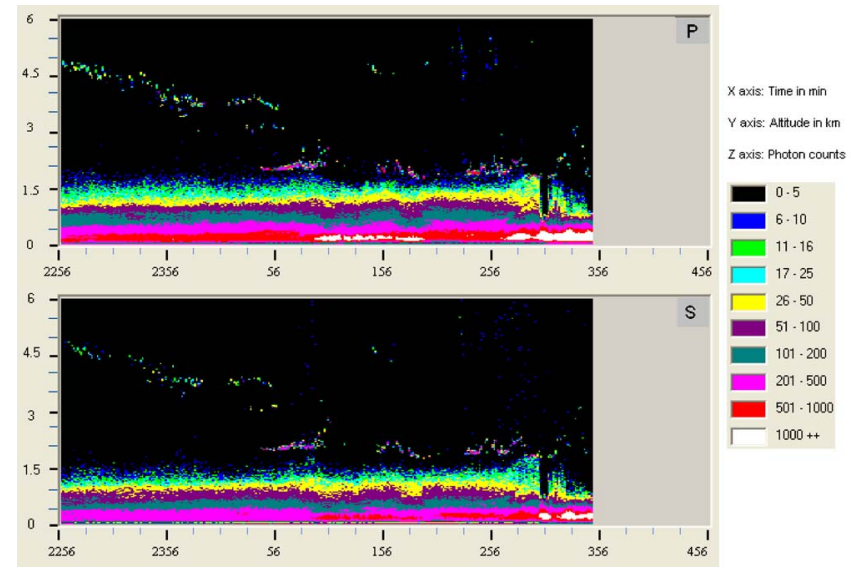

FIG. 9. (Color online) Lidar backscattered raw $P$ and $S$ data acquired on a cloudy day (19 November 2008) during night time.

\section{RESULT AND DISCUSSION}

The fine $S$ beam alignment was carried out by rotating the SMCRM at $0.9^{\circ}$ step. The SMCRM is aligned at the position where optimum $S$ polarization signal reception takes place. Figure 5 shows the typical $S$ polarization signal received during the rotating mirror alignment to its optimum position. As can be seen from the figure, the maximum signal peak and the optimum alignment occurs at step 9 (binary 1001 applied to motor coils). Once the $S$ alignment has been made, the $P$ signal is received by moving the SMCRM anticlockwise by 45 steps.

The dual polarization detection capability of the technique has been tested by acquiring lidar returns and computing depolarization at altitudes starting from $100 \mathrm{~m}$ up to 9 $\mathrm{km}$. Figure 6 shows $P$ and $S$ lidar returns received on 22 January 2009 (on a cloudy day) at 10.50 p.m. local time. The response shows an increased depolarization in the beginning that is in the atmospheric boundary layer at about $600 \mathrm{~m}$. This is particularly due to the nonspherical aerosol particles present at this height. Further rise in depolarization has been observed at the cloud between 5.9 and $7.5 \mathrm{~km}$. A slight delayed peak at the cloud in $S$ signal clearly depicts that this increase in depolarization within the cloud is due to multiple scattering of laser beam in the cloud layer. This effect is more prominent in the second cloud peak detected at $7.3 \mathrm{~km}$.

A further real time backscattered profile acquired on 16 October 2008 night time is shown in Fig. 7. Figure 8 shows the depolarization ratio of the data shown in Fig. 7. Strong depolarization of about $0.3-0.4$ has been observed at the lower altitude. ${ }^{11}$ The depolarization decreases as we move up within the boundary layer. This is due to nonspherical particles at the lower level and relatively more spherical at the higher altitude in this region. A relative low depolarization in the low level cloud at about $1 \mathrm{~km}$ reveals that it contains almost spherical water droplets. ${ }^{12}$

Figures 9 and 10 shows further lidar backscattered and its depolarization acquired on 19 November 2008 during night time, respectively. Thin layer clouds have been observed during this observational period. High depolarization ratio of about 0.7 was observed in the cloud. Along with the 


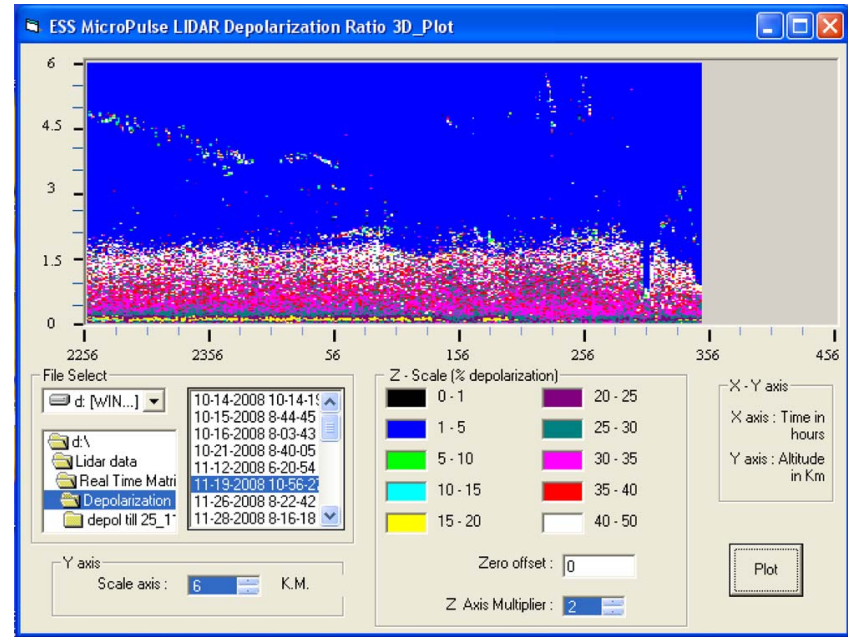

FIG. 10. (Color online) Typical depolarization profile extracted from data acquired on 19 November 2008.

variation of depolarization in the ABL, relatively high depolarization was also observed in the thin cloud layers near to the top of ABL.

Further Fig. 11 shows the high resolution data acquired on 6 January 2009 by the lidar to analyze the variations in the atmospheric boundary layer particles. Along with settlement of aerosol particles at the lower altitude as the nighttime progresses, ${ }^{19,20}$ strong turbulence was also observed at about $1 \mathrm{~km}$, the depolarization of these data is shown in Fig. 12. As can be seen from the figure almost constant depolarization ratio exists in this region from $100 \mathrm{~m}$ to about 1.1 $\mathrm{km}$. However slight increase in $\delta$ can be observed at about $375 \mathrm{~m}$ height (shows high yellow colored picture) which suggests relatively more nonspherical aerosol dust particles in this region.

\section{CONCLUSIONS}

The dual polarization detection technique using single PMT has been successfully developed and tested for its functionality to measure the depolarization ratio in aerosols of the

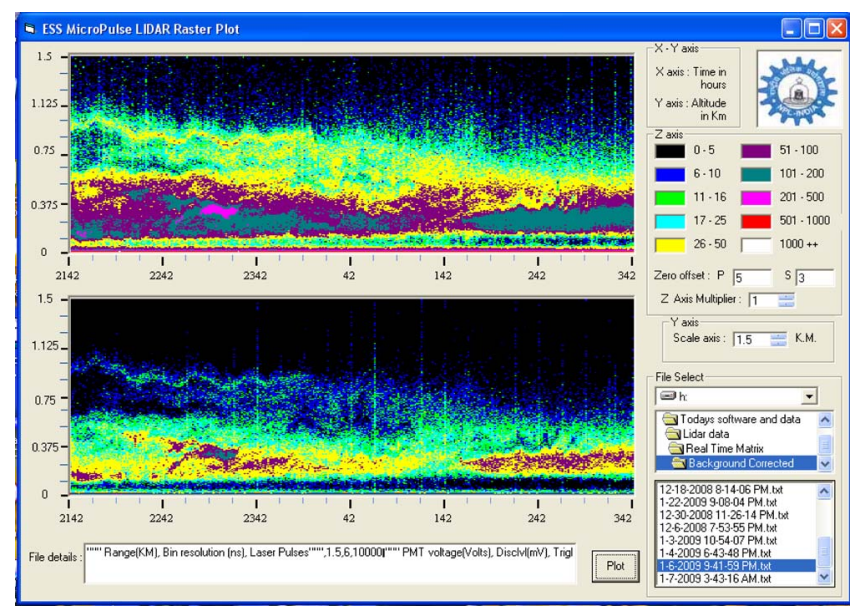

FIG. 11. (Color online) The high resolution $P$ and $S$ data acquired on 6 January 2009 by the lidar to analyze the variations in the atmospheric boundary layer particles

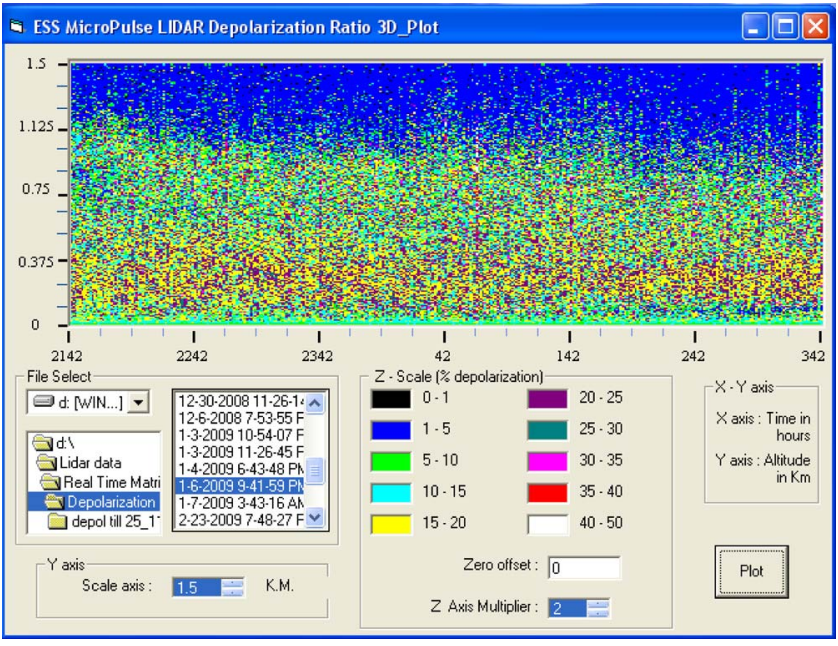

FIG. 12. (Color online) The depolarization ratio of the data acquired on 6 January 2008.

boundary layer and clouds. As the technique is based on the assumption that the atmosphere is stable during the entire $P$ and $S$ acquisition, it requires fast repetition rate pulsed laser and a very high speed photon counting unit. More accurate depolarization ratio may be obtained by using fast enough acquisition electronics.

\section{ACKNOWLEDGMENTS}

Authors are grateful to Director, NPL, and Head RASD for providing the necessary facility and infrastructure to carry out the work. Thanks are also due for the financial support under CSIR emeritus scientist scheme (S. L. Jain and P. K. Dubey) and CSIR fellowship (Pavan S. Kulkarni).

${ }^{1}$ J. D. Spinhirne, IEEE Trans. Geosci. Remote Sens. 31, 48 (1993).

${ }^{2}$ Laser Monitoring of the Atmosphere, edited by E. D. Hinkley (Springer, New York, 1976), Chap. 4.

${ }^{3} \mathrm{U}$. Wandinger, Lidar Range Resolved Optical Remote Sensing of the Atmosphere: Introduction to Lidar, edited by C. Weitkamp (Springer, New York, 2005), p. 1.

${ }^{4}$ M. Shiobara, M. Yabuki, and H. Kobeyashi, Phys. Chem. Earth 28, 1212 (2003).

${ }^{5}$ M. L. Simpson, M.-D. Cheng, T. Q. Dam, K. E. Lenox, J. R. Price, J. M. Storey, E. A. Wachter, and W. G. Fisher, Appl. Opt. 44, 7210 (2005).

${ }^{6}$ R. M. Schotland, K. Sassen, and R. J. Stone, J. Appl. Meteorol. 10, 1011 (1971).

${ }^{7}$ C. J. Flynn, A. Mendoza, Y. Zeng, and S. Mathur, Opt. Express 15, 2785 (2007).

${ }^{8}$ D. M. Winker and M. T. Osborn, Geophys. Res. Lett. 19, 171 (1992).

${ }^{9}$ G. G. Gimmestad, Appl. Opt. 47, 3795 (2008).

${ }^{10}$ A. R. Klekociuk, P. G. Brown, D. W. Pack, D. O. ReVelle, W. N. Edwards, R. E. Spalding, E. Tagliaferri, B. B. Yoo, J. Zagari, Nature (London) 436, 1132 (2008).

${ }^{11}$ C.-W. Chiang, W.-N. Chen, W.-A. Liang, S. K. Das, and J.-B. Nee, Atmos. Environ. 41, 4128 (2007).

${ }^{12}$ See: www.jp.hamamatsu.com, R647-02 PMT datasheet.

${ }^{13}$ P. K. Dubey, S. L. Jain, B. C. Arya, Y. N. Ahammed, A. Arun, D. K. Shukla, and P. S. Kulkarni, Int. J. Remote Sens. (to be published).

${ }^{14}$ See: www.cvilaser.com, PBS-532-050 datasheet.

${ }^{15}$ See: www.jdsu.com, Q201HD laser datasheet.

${ }^{16}$ See: www.picoquant.com, High PRR lasers datasheet.

${ }^{17}$ See: www.crowntech-inc.com, Time Harp 200 photon counting board details.

${ }^{18}$ See: www.fastcomtec.com, model MCS6 datasheet.

${ }^{19}$ Y. B. Kumar, Opt. Eng. 45, 076201 (2006).

${ }^{20}$ R. Endlich, E. Ludwig, and E. Uthe, Atmos. Environ. 13, 1051 (1979). 